\title{
Chapitre 5 Évolution du parc des réacteurs de recherche en France
}

\subsection{Diversité et complémentarité des réacteurs de recherche français}

Dans la base de données de l'AIEA relative aux réacteurs de recherche (Research Reactor Database - RRDB), 42 réacteurs sont recensés pour la France sous l'appellation de réacteur de recherche ${ }^{120}$ ( $\mathrm{y}$ compris ceux qui ne sont plus en exploitation, le réacteur Jules Horowitz $(\mathrm{RJH})$ en cours de construction, ainsi que ceux relevant des installations intéressant la défense nationale $\left.{ }^{121}\right)$.

C'est en 1945 que le Général de Gaulle a créé, par ordonnance, le Commissariat à l'énergie atomique $\left(C E A^{122}\right)$ en le chargeant de diriger et de coordonner le développement des applications de la fission du noyau de l'atome d'uranium. Dans ce contexte, l'équipe dirigée par Lew Kowarski assura le démarrage, en 1948, du premier réacteur de recherche français, la « pile atomique » ZOÉ construite sur le centre du CEA à Fontenayaux-Roses (figure 5.1). Le cœur de ce réacteur, constitué d'éléments combustibles à base d'oxyde d'uranium (1950 kg) plongés dans de l'eau lourde (5 tonnes) contenue dans une

120. Le lecteur pourra trouver la liste complète de ces réacteurs dans cette base de données. Il pourra par ailleurs consulter l'ouvrage du CEA « Les réacteurs nucléaires expérimentaux », monographie de la Direction de l'énergie nucléaire - 2012, ou encore la publication « Les réacteurs de recherche » de Francis Merchie, Encyclopédie de l'énergie, 2015.

121. Réacteurs qui ne sont pas traités dans le cadre du présent ouvrage.

122. Qui deviendra ultérieurement le Commissariat à l'énergie atomique et aux énergies alternatives. 


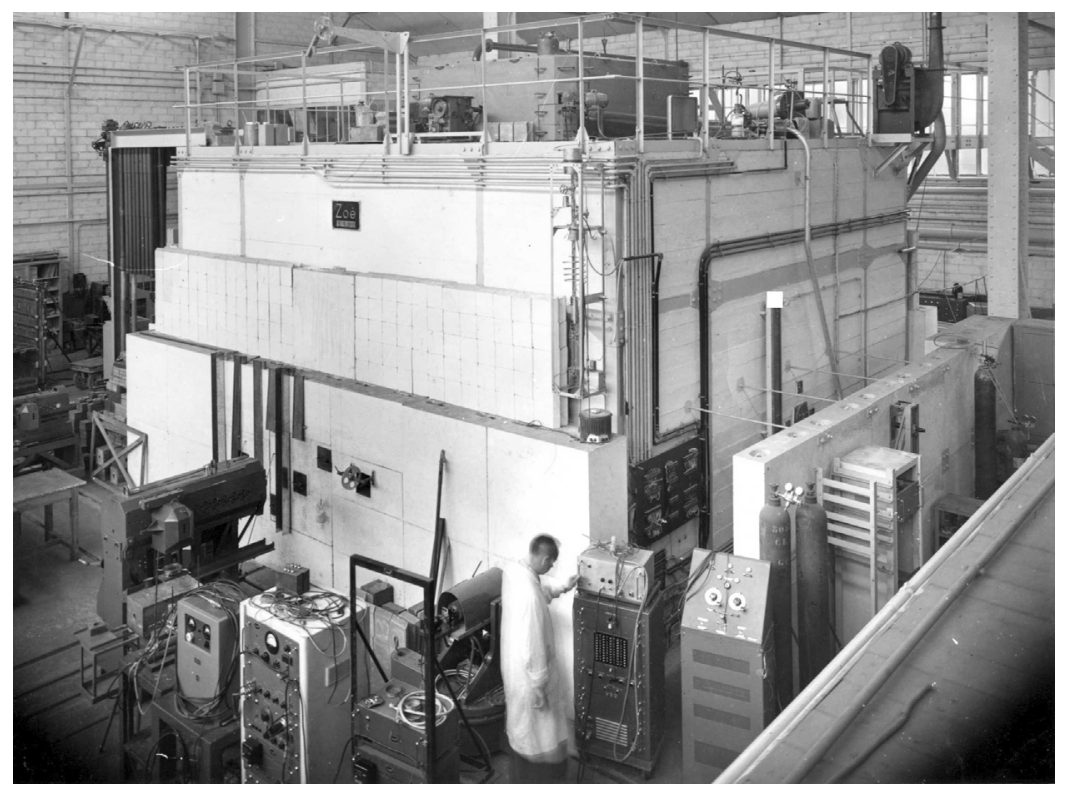

Figure 5.1. Vue de ZOÉ, première « pile atomique » française. Archives historiques CEA. C CEA/Service de documentation.

cuve en aluminium entourée d'un mur de graphite de $90 \mathrm{~cm}$ d'épaisseur, était entouré d'une enceinte en béton de 1,5 mètre d'épaisseur destinée à absorber les différents types de rayonnements ionisants émis par les réactions nucléaires dans le cœur. Le réacteur ZOÉ fut utilisé jusqu'à une puissance de $150 \mathrm{~kW}$ pour étudier le comportement de matériaux sous irradiation et à faible puissance, pour caractériser les propriétés neutroniques des matériaux constitutifs des piles atomiques de l'époque (sur le plan mondial).

Dans les années 1950, une dizaine de réacteurs de recherche furent mis en service en France. Ne disposant pas à l'époque d'une capacité d'enrichissement industrielle propre, la France s'est alors engagée dans l'amélioration de la connaissance des données nucléaires pour les réacteurs utilisant de l'uranium naturel. Le réacteur AQUILON, implanté à Saclay, fut conçu dans cet objectif. Ce réacteur, ainsi que le réacteur ALIZÉ (également implanté à Saclay), ont ensuite été utilisés en support à la conception des réacteurs embarqués des premiers sous-marins français à propulsion nucléaire. Le réacteur PROSERPINE, également implanté à Saclay, fut dédié à l'étude de réacteurs dits « homogène ${ }^{123}$ " utilisant du plutonium en solution comme matière fissile ; il était modéré à l'eau légère. Ce réacteur a permis de comparer les caractéristiques neutroniques de deux éléments fissiles fondamentaux : le plutonium 239 et l'uranium 235.

En parallèle, toujours durant les années 1950, des réacteurs d'irradiation et de recherche technologique furent construits. C'est ainsi que furent mis en service à Saclay le réacteur EL2 puis le réacteur EL3, avec pour objectif, d'une part la production de

123. Le combustible d'un réacteur homogène est sous forme liquide (nitrate ou sulfate). 
radioisotopes artificiels, d'autre part l'étude du comportement sous irradiation de matériaux de structure des réacteurs.

Vers la fin des années 1950, il apparut nécessaire d'affiner les connaissances relatives aux paramètres neutroniques fondamentaux intervenant dans la physique des cœurs de réacteurs nucléaires. C'est pour répondre notamment à ce besoin que le réacteur MINERVE fut conçu et mis en service en 1959 au centre du CEA de Fontenay-aux-Roses.

Les années 1960 ont ensuite vu la mise en service d'une vingtaine de réacteurs de recherche. À cette époque, le développement de filières industrielles de l'énergie nucléaire était pleinement engagé mais les moyens de calcul disponibles étaient limités. $L^{\prime}$ 'usage des maquettes ou assemblages critiques ${ }^{124}$ et des réacteurs d'irradiation est alors apparu nécessaire pour poursuivre et compléter l'acquisition de connaissances et de données en support au développement industriel de l'énergie nucléaire. La France cherchait alors à développer la filière électronucléaire dite UNGG (uranium naturelgraphite-gaz) utilisant l'uranium naturel comme combustible. Les réacteurs de recherche MARIUS (mis en service en 1960 sur le site de Marcoule puis transféré au milieu des années 1960 sur le site CEA de Cadarache) et CESAR (mis en service en 1964 à Cadarache) ont servi, au début des années 1960, à des études pour cette filière.

L'utilisation de réacteurs à neutrons rapides (RNR) a également été explorée dès cette époque, notamment dans l'objectif de valoriser les ressources en plutonium résultant du fonctionnement des réacteurs UNGG. Le développement des RNR a en particulier conduit à la construction :

- du réacteur HARMONIE, implanté à Cadarache et dont la première divergence est intervenue en 1965, qui a été utilisé essentiellement pour la détermination des caractéristiques neutroniques de matériaux de protection radiologique (protections neutroniques latérales situées autour du cœur dans les RNR) ;

- de la maquette critique MASURCA ${ }^{125}$, également implantée à Cadarache et mise en service en 1966, qui a permis de réaliser des études de neutronique ainsi que, bien plus tard, des recherches dans le domaine de la transmutation des actinides présents dans les déchets nucléaires fortement radioactifs.

Le réacteur RAPSODIE est le premier réacteur à neutrons rapides ayant servi à des recherches et ayant fonctionné à la fois avec du plutonium ${ }^{126}$ comme combustible et du sodium liquide comme fluide caloporteur. De nombreuses expériences d'irradiation y ont été menées, de 1967 (année de sa première divergence) à 1982 (arrêt définitif en 1983), dans le cadre du développement d'aciers de gainage pour les RNR. Des expérimentations dites « essais de fin de vie », allant jusqu'à la fusion de combustible au cœur de certaines aiguilles de combustible, ont été réalisées en 1982 (essais DISCO et FONDU).

124. Réacteurs mettant en œuvre des arrangements d'éléments combustibles représentatifs des cœurs à étudier (" maquette ») et fonctionnant à une puissance quasi nulle (état « juste critique »).

125. Maquette de surgénération du centre d'études de Cadarache.

126. Le réacteur RAPSODIE a utilisé, comme les RNR français suivants, de l'oxyde mixte $\cup_{2}-\mathrm{PuO}_{2}$; étaient aussi implantées, autour de la zone fissile, des « couvertures » axiales et radiales en uranium 238 (appauvri en isotope 235), matériau fertile sous flux de neutrons rapides. 
Le réacteur $C A B R I$, premier réacteur français dédié à la réalisation d'essais pour l'étude des situations accidentelles des RNR (dans une boucle en sodium), a été construit à Cadarache au début des années 1960 ; la première divergence de ce réacteur sera réalisée au mois de décembre 1963. Vont également y être menés, dans la boucle en sodium, des essais pour l'étude de situations accidentelles dans les réacteurs à eau sous pression (essais dits REP-Na). Le réacteur SCARABEE, utilisé dans les années 1980 pour des essais relatifs aux réacteurs à neutrons rapides refroidis par du sodium (arrêté depuis et démonté), partageait les principaux équipements du réacteur CABRI. Il était équipé d'une boucle en sodium de plus grand diamètre que celle utilisée dans le réacteur CABRI.

La mise à disposition par les États-Unis d'uranium fortement enrichi en isotope 235 a permis de concevoir, dans les années 1960, des cœurs de réacteurs délivrant des puissances et des flux neutroniques accrus, qui en feront des réacteurs d'irradiations technologiques. En France, trois réacteurs d'irradiation ont été conçus à cette époque : le réacteur PEGASE, d'une puissance de $30 \mathrm{MW}$, implanté à Cadarache, le réacteur SILOE, d'une puissance de $35 \mathrm{MW}$, implanté à Grenoble (circulation forcée de l'eau en sens descendant ; ce réacteur a fonctionné de 1963 à 1997), et le réacteur OSIRIS (figure 5.2), d'une puissance de $70 \mathrm{MW}$, implanté à Saclay (circulation en sens ascendant ; a fonctionné de 1966 à 2015). Ces réacteurs ont été accompagnés de maquettes critiques : PEGGY pour PEGASE, SILOETTE (figure 5.2) pour SILOE et ISIS pour OSIRIS.
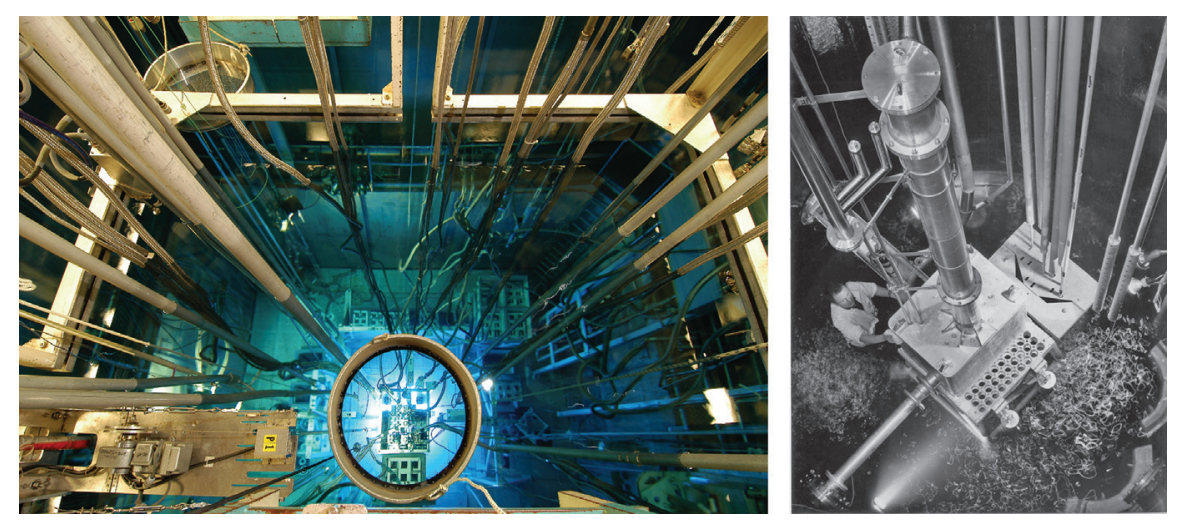

Figure 5.2. À gauche, cœur du réacteur Osiris. Vue de l'installation immergée d'examen de neutronographie (2004). (c) L. Godart/CEA ; à droite vue de la maquette SILOETTE. () CEA.

Contrairement au réacteur à haut flux (RHF) à Grenoble et au réacteur ORPHEE, SILOE était un réacteur de type piscine à eau légère construit pour l'irradiation des matériaux et des dispositifs. Le cœur (figure 5.3) était constitué d'éléments dont le combustible était enrichi à $90 \%$ en uranium 235.

Le réacteur SILOE s'est néanmoins vu doté de canaux neutroniques ne visant pas directement le cœur, ainsi que d'un mur de béryllium ${ }^{127}$ qui jouxtait l'un des quatre côtés

127. Ce matériau est une source de neutrons lorsqu'il est frappé notamment par des rayons gamma de forte énergie. 


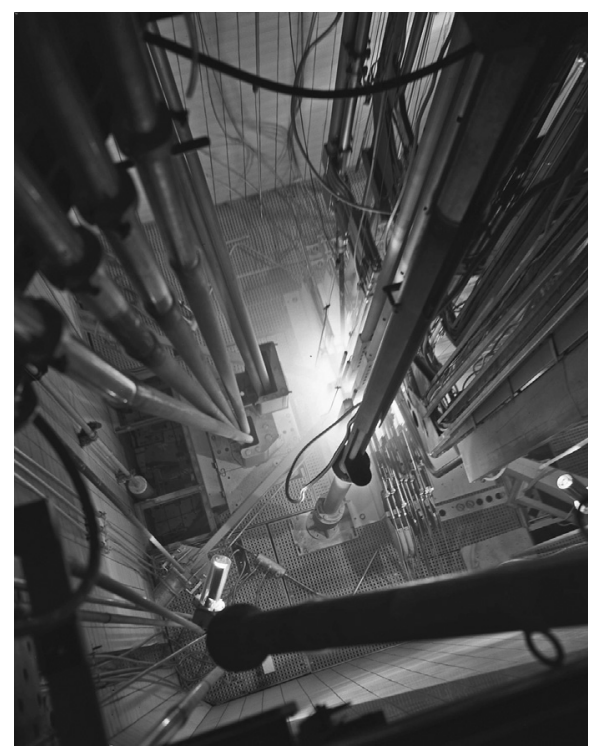

Figure 5.3. Vue du cœur du réacteur SILOE. (C) CEA.

du cœur ${ }^{128}$. Au début, il y avait seulement deux canaux radiaux. Après la fermeture du réacteur MELUSINE en 1988, un canal tangentiel a été ajouté dans SILOE qui visait le mur de béryllium par la tranche. Le parc d'instruments est alors monté à six avec deux appareils par canal (spectromètres, diffractomètres). Malgré des conditions difficiles de travail pour les scientifiques (température élevée, espaces restreints...), ces équipements ont pu rendre d'appréciables services au plan scientifique et ont permis de former des scientifiques, notamment en diffraction de poudres et de monocristaux, ainsi qu'en diffraction des neutrons polarisés.

En 1969, la France prend la décision d'abandonner la réalisation de réacteurs UNGG au profit des réacteurs à eau sous pression de conception américaine. Dans la décennie qui suit, les besoins expérimentaux étant largement satisfaits, peu de nouveaux réacteurs de recherche seront construits en comparaison de la période 1950-1970.

Cependant, deux réacteurs à flux élevé de neutrons, équipés de canaux neutroniques pour des expériences de physique fondamentale, ont ensuite été mis en service : il s'agit du réacteur à haut flux (RHF, de $58 \mathrm{MW}$ ) implanté à Grenoble, de l'Institut Laue-Langevin (ILL), qui divergea en 1971, ainsi que du réacteur ORPHEE (14 MW) implanté à Saclay, qui divergea à la fin de l'année 1980.

Il est à noter qu'en 1972 le CEA créa, en association avec EDF, le Département de construction des piles, au sein de la Direction des piles atomiques du CEA, qui sera ensuite

128. Source : Association des retraités de l'Institut Laue-Langevin (ARILL) : « Le réacteur de recherche SILOE ». 
externalisé et rattaché à la société Technicatome ${ }^{129}$ puis constituera la branche TA de la société AREVA. Ces entités et sociétés contribueront de façon importante à la conception et à la construction de réacteurs de recherche, jusques et y compris le réacteur Jules Horowitz.

Pour ce qui concerne le développement des RNR, un nombre important d'irradiations expérimentales ont été menées dans le réacteur à vocation électrogène PHENIX, mis en service en 1974, jusqu'à son arrêt définitif au mois de février 2010. Comme dans le cas du réacteur RAPSODIE, des essais ultimes dits aussi « de fin de vie ${ }^{130}$ » ont été réalisés en rapport avec certaines questions de sûreté (par exemple, la possibilité d'amorçage d'une convection naturelle du sodium dans différents circuits du réacteur, le risque de rupture de gaine en cas de fusion partielle à cœur dans une aiguille de pastilles de combustible...).

De même, le réacteur d'essais PHEBUS a été mis en service en 1978 à Cadarache pour l'étude des accidents de refroidissement des réacteurs à eau sous pression.

Enfin, le CEA lance le projet d'un nouveau réacteur d'irradiations technologiques, le réacteur Jules Horowitz. En cours de construction à Cadarache, il prendra la suite du réacteur OSIRIS dont l'arrêt définitif est intervenu à la fin de l'année 2015. Nous reviendrons plus en détail sur ce nouveau réacteur par la suite.

Le tableau 5.1 ci-après présente les réacteurs français utilisés en tout ou partie pour des expérimentations, avec leur date de mise en service (hors installations de même nature intéressant la défense nationale).

\subsection{Situation actuelle}

Depuis la pile ZOÉ, une quarantaine de réacteurs de recherche ont donc été construits et exploités en France. Mi 2018, avec l'arrêt du réacteur OSIRIS, sept réacteurs de recherche restent « en fonctionnement ${ }^{131}$ ", les autres étant promis à l'arrêt définitif sans utilisation (PHEBUS), en phase de cessation définitive d'activité, en cours de démantèlement ou déjà démantelés.

Parmi les réacteurs en fonctionnement, trois sont des maquettes critiques : EOLE, MINERVE et MASURCA, toutes implantées aujourd'hui à Cadarache. Le réacteur MINERVE (figure 5.4), mis en service en 1959 sur le centre de Fontenay-aux-Roses a été transféré à Cadarache en 1977. Il est dédié à la caractérisation neutronique de matériaux (matériaux fissiles, fertiles, absorbants ou matériaux de structures) et à l'étude de la physique des cœurs de réacteurs de différentes filières. Il s'agit d'un réacteur de type

129. La société GAAA (Groupement atomique alsacienne atlantique) devenue ensuite Novatome s'impliquant en association avec le CEA et EDF dans la conception des réacteurs à neutrons rapides.

130. Voir l'ouvrage de Joël Guidez « PHENIX - Le retour d'expérience », chapitre XV, Collection : Hors collection, EDP sciences, 2013.

131. ORPHEE, ISIS, MASURCA, CABRI, EOLE, MINERVE et le RHF. La situation concernant EOLE et MINERVE est néanmoins évoquée plus loin. 
Tableau 5.1. Liste des réacteurs français utilisés en tout ou partie pour des expérimentations, avec leur date de mise en service.

\begin{tabular}{|c|c|c|c|c|}
\hline $\begin{array}{l}\text { Type de } \\
\text { réacteur } \rightarrow \\
\text { Utilisations } \\
\downarrow\end{array}$ & $\begin{array}{c}\text { Réacteurs utilisant } \\
\text { de l'eau (légère ou } \\
\text { lourde) ou de l'air } \\
\text { comme fluide } \\
\text { caloporteur dans le } \\
\text { cœur }\end{array}$ & $\begin{array}{l}\text { Réacteurs } \\
\text { utilisant du } \\
\text { sodium } \\
\text { comme fluide } \\
\text { caloporteur } \\
\text { dans le cœur }\end{array}$ & $\begin{array}{l}\text { Maquettes } \\
\text { critiques }\end{array}$ & $\begin{array}{l}\text { Réacteurs à } \\
\text { faisceaux } \\
\text { sortis de } \\
\text { neutrons (eau } \\
\text { lourde/eau } \\
\text { légère) }\end{array}$ \\
\hline $\begin{array}{l}\text { Études de } \\
\text { physique des } \\
\text { réacteurs } \\
\text { (y compris de } \\
\text { détermination } \\
\text { de données } \\
\text { nucléaires), de } \\
\text { protections } \\
\text { radiologiques } \\
\text { d'irradiation, } \\
\text { pour tous } \\
\text { usages }\end{array}$ & $\begin{array}{l}\text { ZOE (1948) } \\
\text { EL2 (1952) } \\
\text { EL3 (1957) } \\
\text { MELUSINE (1959) } \\
\text { TRITON (1959) } \\
\text { NEREIDE (1960) } \\
\text { PEGASE (1963) } \\
\text { SILOE (1963) } \\
\text { OSIRIS (1966) } \\
\text { RJH (en construction) }\end{array}$ & $\begin{array}{l}\text { RAPSODIE (1966) } \\
\text { PHÉNIX (1974) }\end{array}$ & $\begin{array}{l}\text { AQUILON (1956) } \\
\text { PROSERPINE (1958) } \\
\text { ALIZE (1959) } \\
\text { RUBEOLE (1959) } \\
\text { MINERVE (1959) } \\
\text { MARIUS (1960) } \\
\text { PEGGY (1961) } \\
\text { RACHEL (1961) } \\
\text { SILOETTE (1964) } \\
\text { CESAR (1964) } \\
\text { EOLE (1965) } \\
\text { ISIS (1966) } \\
\text { HARMONIE (1965) } \\
\text { MASURCA (1966) }\end{array}$ & \\
\hline $\begin{array}{l}\text { Essais de } \\
\text { sûreté }\end{array}$ & $\begin{array}{l}\text { CABRI (boucle en } \\
\text { sodium 1972) } \\
\text { PHEBUS (1978) } \\
\text { CABRI (boucle en eau) }\end{array}$ & $\begin{array}{l}\text { RAPSODIE (1966) } \\
\text { PHÉNIX (1973) } \\
\text { (pour les essais } \\
\text { « de fin de vie ») }\end{array}$ & & \\
\hline $\begin{array}{l}\text { Études de la } \\
\text { physique de la } \\
\text { matière }\end{array}$ & & & & $\begin{array}{l}\text { RHF (1971) } \\
\text { ORPHEE (1980) }\end{array}$ \\
\hline Enseignement & $\begin{array}{l}\text { MINERVE (1959) } \\
\text { ULYSSE (1961) type } \\
\text { Argonaute } \\
\text { SILOETTE (1964) } \\
\text { RUS « Cronenbourg » } \\
\text { (1966) type Argonaute } \\
\text { ISIS (1966) }\end{array}$ & & & \\
\hline
\end{tabular}

piscine, dont la puissance maximale de fonctionnement est de $100 \mathrm{~W}$. Le cœur du réacteur, constitué d'éléments combustibles sous la forme de plaques à base d'alliage UAl, enrichi à environ $93 \%$ en uranium 235 , est entouré d'éléments réflecteurs en graphite et immergé dans un grand volume d'eau déminéralisée $\left(140 \mathrm{~m}^{3}\right)$, qui permet d'assurer, d'une part la protection des opérateurs contre les rayonnements ionisants, d'autre part l'évacuation de la (faible) puissance thermique du cœur. 


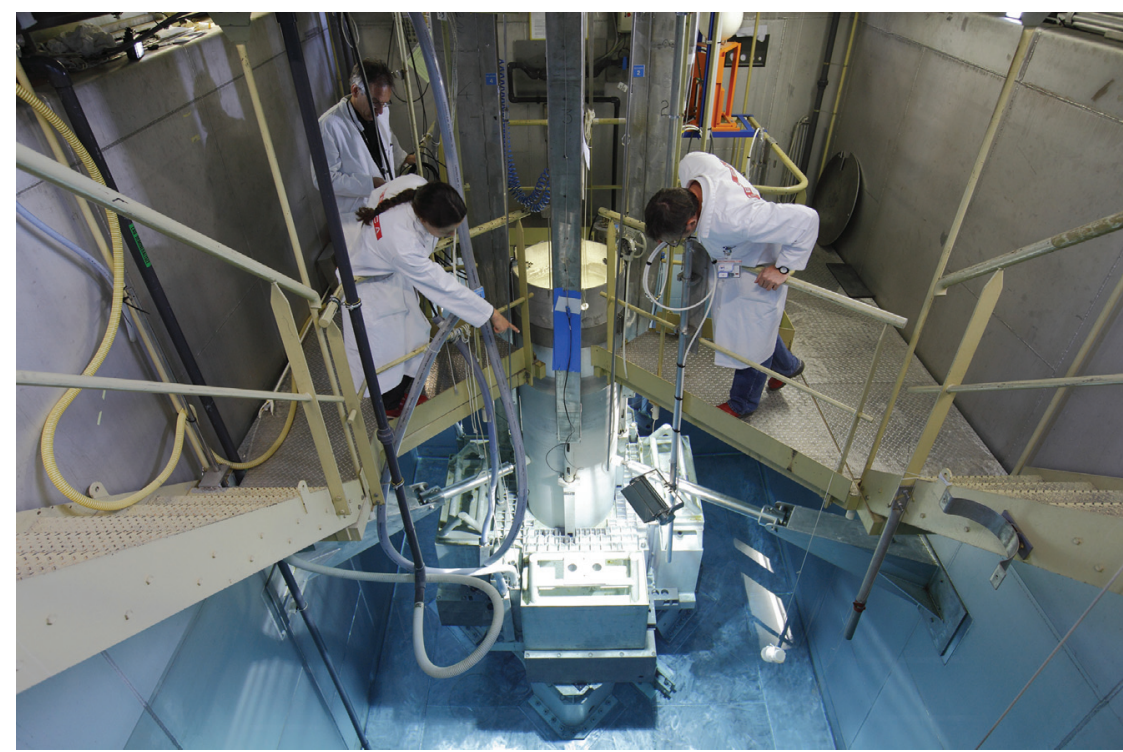

Figure 5.4. La piscine de MINERVE. Chargement d'un échantillon dans la cavité centrale pour la mesure d'effet en réactivité par la méthode dite d'oscillation. (C) G. Lesénéchal/CEA.

Le réacteur EOLE (figure 5.5), mis en service en 1965, est implanté dans le même bâtiment que le réacteur MINERVE. Il fonctionne également à une très faible puissance (100 W au maximum), mais sa conception est très différente de celle du réacteur MINERVE. Le réacteur EOLE, auquel sont associés différentes moyens de mesure (spectrométrie $\gamma$, chambres à fission, détecteurs thermoluminescents), permet de caractériser entièrement des configurations de cœur d'un point de vue physique et neutronique. Lors de chaque programme expérimental réalisé dans le réacteur EOLE, un nouveau cœur est mis en place à partir des éléments de combustible disponibles de l'installation. Ce cœur est placé dans une cuve métallique de taille réduite (environ 1 mètre de hauteur et 1 mètre de diamètre) à l'intérieur de laquelle de l'eau, maintenue en température et éventuellement borée, est introduite progressivement jusqu'à l'atteinte de la criticité. Des mesures sont alors effectuées sur les éléments combustibles non seulement en puissance mais également après l'arrêt du réacteur. En répétant ces mesures pour différentes configurations du cœur expérimental (changement d'un matériau, remplacement d'un élément combustible par de l'eau, insertion d'un dispositif expérimental, etc.), il est possible non seulement de caractériser les effets des matériaux et des dispositifs testés sur la réactivité du coeur, mais également d'évaluer des paramètres neutroniques caractéristiques tels que l'effet Doppler ou l'effet du modérateur (contre-réactions neutroniques). Le réacteur EOLE a par exemple contribué à la qualification d'outils de modélisation neutronique utilisés pour la conception du réacteur de recherche Jules Horowitz (RJH).

La maquette critique MASURCA (figure 5.6), également implantée à Cadarache, a la particularité de ne pas être refroidie par de l'eau mais par de l'air. Les cœurs expérimentaux qui y sont chargés sont bien plus imposants (jusqu'à $6 \mathrm{~m}^{3}$ de volume) que ceux 


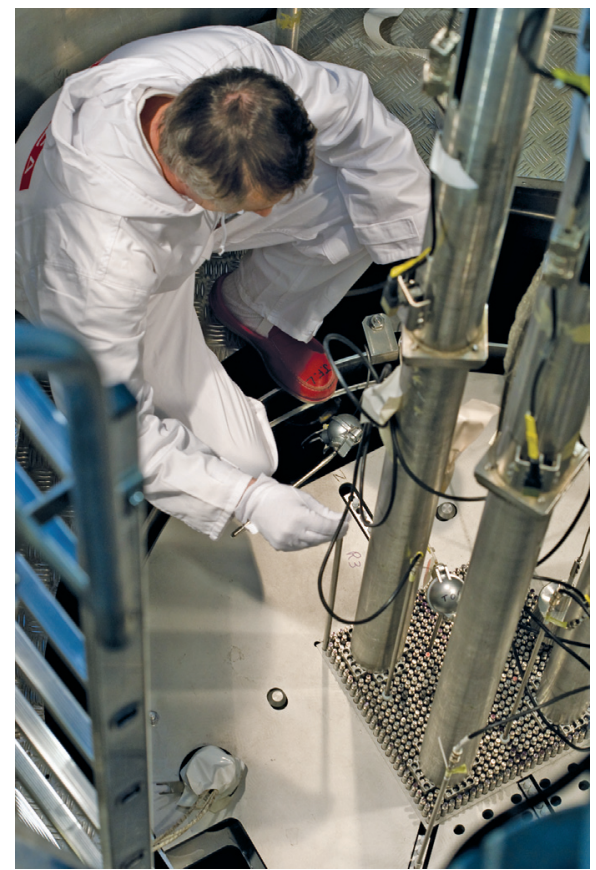

Figure 5.5. Vue de la cuve du réacteur EOLE configurée pour l'expérience PERLE (2008). @ P. Dumas/CEA.

qui peuvent être chargés dans les réacteurs EOLE ou MINERVE. La maquette MASURCA, dont la puissance maximale est de $5 \mathrm{~kW}$, a été spécifiquement conçue pour l'étude des caractéristiques des cœurs des réacteurs à neutrons rapides. Chaque cœur expérimental est constitué de tubes de section carrée, remplis à la main de petits

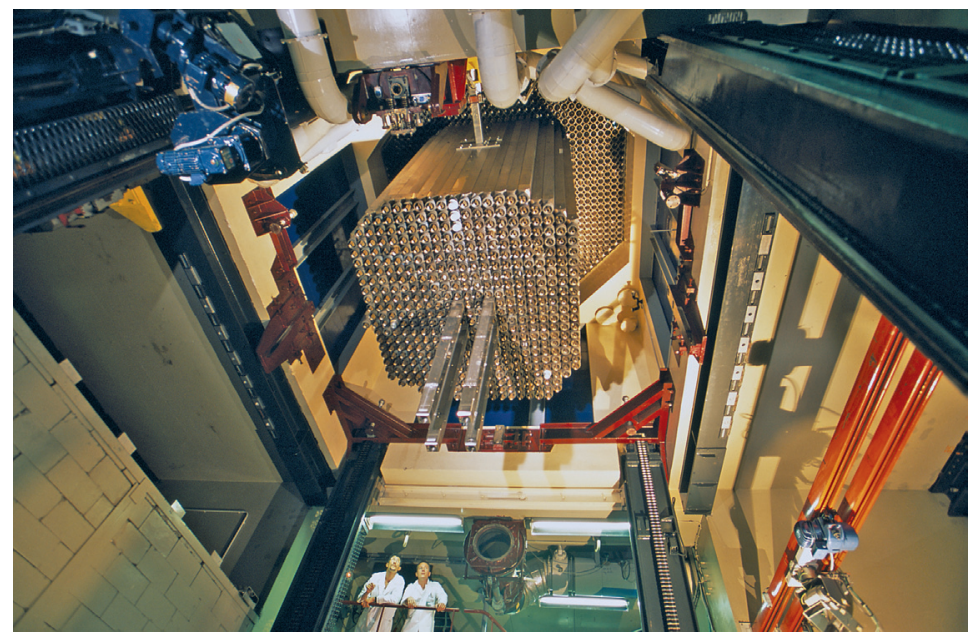

Figure 5.6. Réacteur MASURCA : vue de dessous du cœur, descente d'éléments de combustible. (c) P. Stroppa/CEA. 
éléments combustibles (sous forme de réglettes ou de plaquettes), d'éléments absorbants ou d'éléments de caloporteur (sodium sous forme solide) afin de représenter le réseau à étudier au plan neutronique. Cette possibilité de constitution de cœurs « à la carte » permet d'étudier des concepts de cœurs de réacteurs très variés et novateurs, tels que les réacteurs hybrides sous-critiques (ADS en anglais, pour Accelerator Driven System) ou les réacteurs à haute température (HTR en anglais, pour High Temperature Reactor). La maquette MASURCA est à l'arrêt depuis 2007 le CEA ayant l'intention d'y mener d'importants travaux de rénovation pour répondre à des questions de neutronique relatives à d'éventuels réacteurs à neutrons rapides dits de quatrième génération, en particulier pour le projet ASTRID (Advanced Sodium Technological Reactor for Industrial Demonstration).

Si le réacteur MASURCA est maintenu en exploitation les expériences dans les réacteurs EOLE et MINERVE ont été arrêtées fin 2017 ; une installation, dénommée ZEPHYR, est en projet pour leur succéder.

Aux réacteurs de recherche de faible puissance peuvent être rattachés les réacteurs utilisés pour l'enseignement. Ils servent à la réalisation des programmes pédagogiques des établissements d'enseignement spécialisés dans le domaine nucléaire, ainsi que des programmes de formation des exploitants et d'organismes de sûreté. Dans le cadre de ces programmes, les réacteurs utilisés sont, soit des réacteurs dédiés de manière quasi exclusive à la formation (réacteur ISIS à Saclay), soit des réacteurs dont le fonctionnement intègre des périodes de formation (réacteur MINERVE à Cadarache).

Le réacteur ISIS (figure 5.7) a été conçu comme une maquette neutronique à l'échelle $1 / 10^{e}$ du réacteur OSIRIS. D'une puissance maximale de $700 \mathrm{~kW}$, ce réacteur a été utilisé pour effectuer des essais relatifs à de nouvelles configurations du cœur d'OSIRIS, ainsi qu'à de nouveaux combustibles ou de nouvelles expériences d'irradiation dans ce réacteur. Une importante rénovation du réacteur ISIS a été réalisée entre 2004 et 2006 afin d'adapter pleinement ce réacteur à la formation. En particulier, la salle de conduite a été aménagée pour pouvoir accueillir les participants à des sessions de formation. Un logiciel de supervision a été développé, qui permet de suivre l'évolution des paramètres pertinents lors de chaque manipulation dans le réacteur. Cette installation, désormais affectée de façon exclusive à la formation, réalise en moyenne une centaine de séances de travaux pratiques d'une demi-journée par an ; son arrêt est prévu en 2019.

En matière d'irradiations technologiques ${ }^{132}$, la France a notamment utilisé (jusqu'en 2015) le réacteur OSIRIS implanté à Saclay. Les activités correspondantes seront reprises par le réacteur Jules Horowitz, en construction à Cadarache, mais dont la mise en service n'est pas envisagée avant $2020^{133}$.

Il est rappelé que les réacteurs d'irradiations technologiques ont été conçus pour produire des flux relativement élevés de neutrons et accueillir des dispositifs, insérés dans le cœur du réacteur ou placés à sa périphérie, dans lesquels les éléments à irradier sont placés.

132. Materials Testing Reactors en anglais.

133. "Le chantier du réacteur Jules Horowitz », Libération $\left\{\right.$ Sciences $\left.^{2}\right\}, 25$ janvier 2016. 

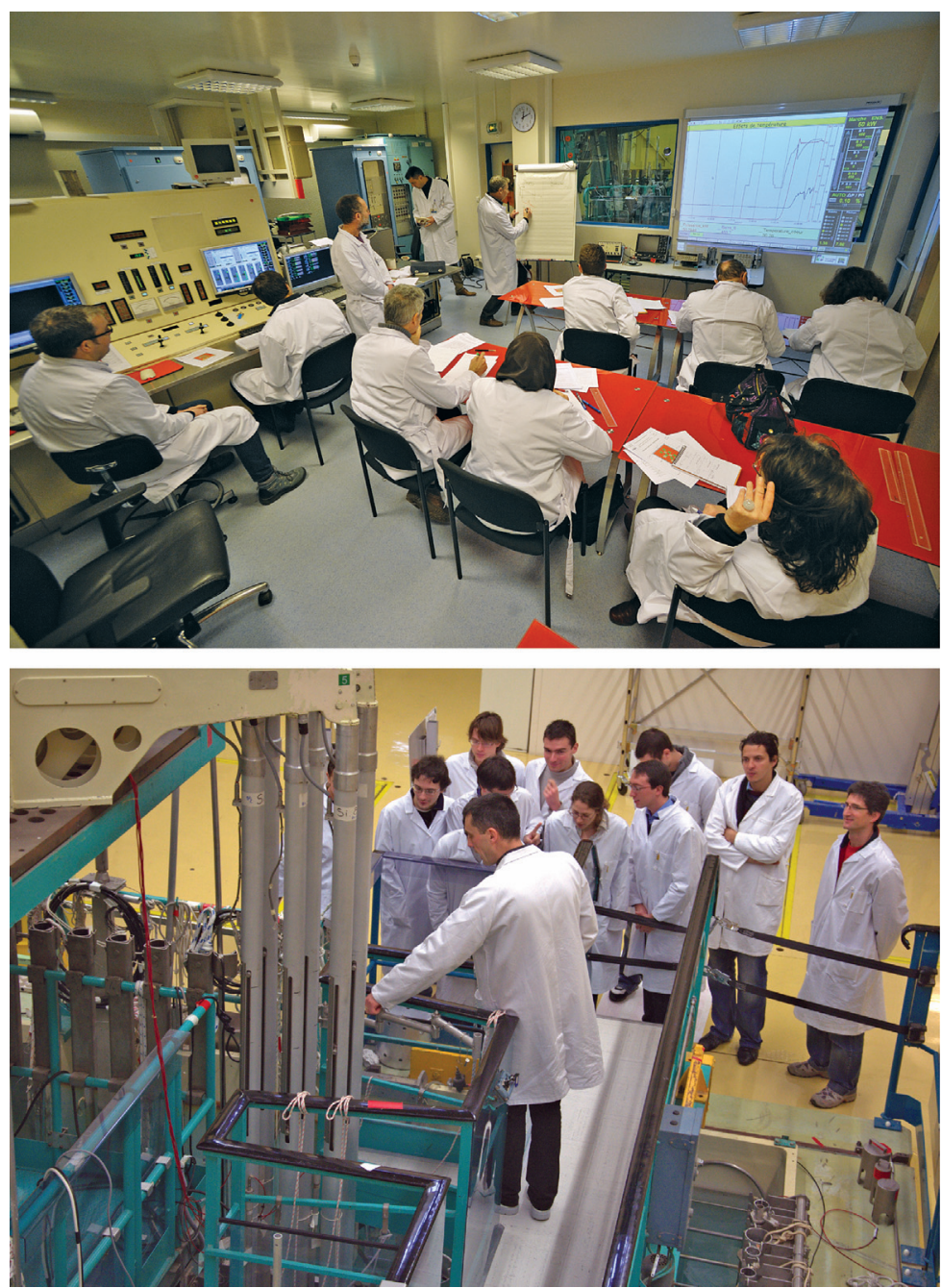

Figure 5.7. En haut, Master français, travaux pratiques en salle de conduite du réacteur ISIS (2013). (C) PF. Grosjean/CEA. En bas, travaux pratiques dans le réacteur ISIS ; élèves de l'École des Ponts et chaussées, cursus « Principes et opérations sur les réacteurs nucléaires » (2010). ๑ S. Renard/CEA.

Le réacteur OSIRIS (figure 5.8), mis en service en 1966, était de type piscine à cœur ouvert. L'eau y servait à la fois de modérateur ${ }^{134}$, de caloporteur ${ }^{135}$ et de protection radiologique. Son cœur, de dimensions réduites $(57 \mathrm{~cm} \times 57 \mathrm{~cm} \times 60 \mathrm{~cm})$, comportait 44 éléments combustibles et pouvait dégager une puissance maximale de $70 \mathrm{MW}$. L'eau circulait de bas en haut dans le cœur. Les flux importants de neutrons, que ce soit à

134. Un modérateur est un matériau qui ralentit les neutrons par chocs successifs de ceux-ci sur des atomes (généralement d'hydrogène) présents dans ce matériau.

135. Un caloporteur absorbe et évacue la chaleur produite dans le combustible par les réactions de fission. 


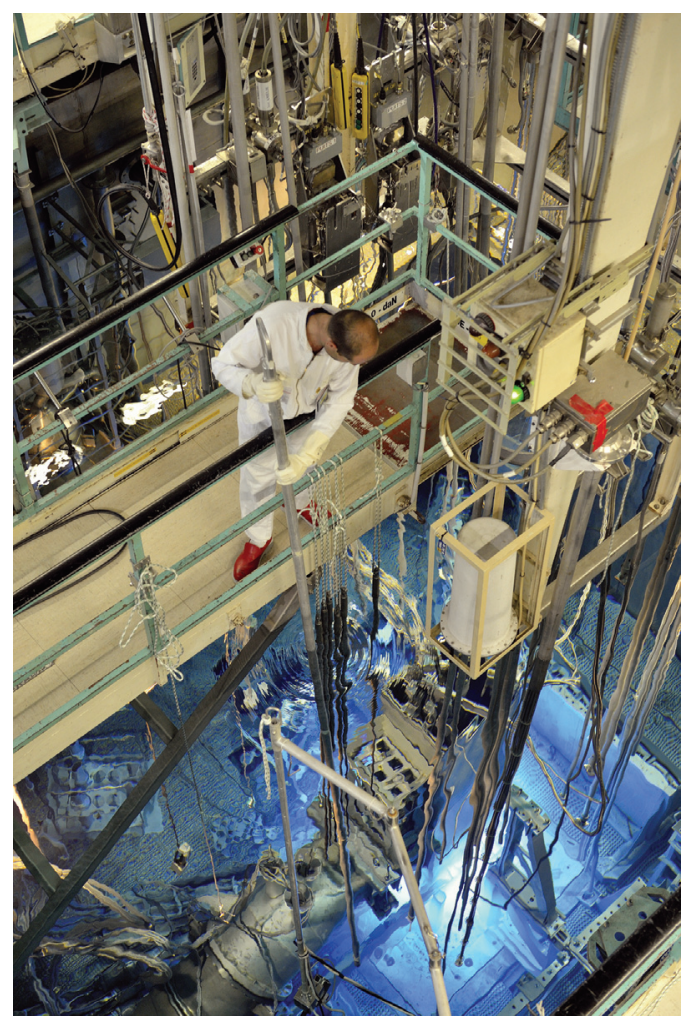

Figure 5.8. Piscine du réacteur OSIRIS. @ Laurent Zylberman/Graphix-Images/IRSN.

l'intérieur du cœur ou à sa périphérie, étaient supérieurs à ceux qui existent dans un réacteur de puissance à eau sous pression, permettant l'étude du vieillissement accéléré de matériaux sous irradiation de ces réacteurs. Ils permettaient aussi la production de radioisotopes artificiels utilisés en médecine pour le diagnostic par scintigraphie ou pour le traitement de certains cancers (curiethérapie) ou d'autres pathologies. Enfin, l'irradiation de lingots monocristallins de silicium permettait une modification de la structure atomique de ce matériau, qui lui confère des propriétés de semi-conducteur (le silicium ainsi « dopé » est utilisé par l'industrie électronique).

\section{Un réacteur d'irradiations de nouvelle génération : le réacteur Jules Horowitz}

Le réacteur Jules Horowitz ${ }^{136}$, ou RJH, dont les études de conception ont commencé en 1998 au CEA, devrait répondre aux besoins exprimés par la Commission européenne de

136. Sources utilisées : « Les réacteurs nucléaires expérimentaux », monographie de la Direction de l'énergie nucléaire - 2012, pages 95 à 106, « Le RJH » site internet CEA Cadarache (http://www. cea.fr/Pages/domaines-recherche/energies/energie-nucleaire/reacteur-de-recherche-jules-horowitz-RJH.aspx), qui met notamment en lien le dossier rendu public sur le site de l'ASN « Réacteur Jules Horowitz - Évaluation complémentaire de la sûreté au regard de l'accident survenu à la centrale de Fukushima I » (CEA/DEN/CAD/DIR/CSN DO 575 13/09/11), l'article Wikipédia et ses références... 
disposer d'un outil de recherche moderne et flexible - les autres réacteurs européens pouvant assurer les mêmes fonctionnalités datant des années 1960 (voir le tableau 5.2 ci-après) - pour y réaliser des expériences (une vingtaine simultanément) contribuant à :

- l'amélioration de la compétitivité et la durée de vie des réacteurs nucléaires de puissance en fonctionnement,

- le développement des performances des combustibles nucléaires des réacteurs dits de « troisième génération » comme l'EPR (European Pressurized Reactor ${ }^{137}$ ),

- le développement de nouveaux matériaux et combustibles pour les réacteurs dits de « quatrième génération » comme celui du projet ASTRID,

- la fiabilisation de la fourniture en Europe de radioisotopes pour le secteur médical.

Tableau 5.2. Réacteurs d'irradiation en Europe (source CEA).

\begin{tabular}{|c|c|c|c|}
\hline Pays & $\begin{array}{c}\text { Réacteur de } \\
\text { recherche }\end{array}$ & Âge (en 2018) & $\begin{array}{c}\text { Puissance } \\
\text { (MWth) }\end{array}$ \\
\hline Belgique & BR2 à Mol & 55 & 60 \\
\hline Pays-Bas & HFR à Petten & 57 & 45 \\
\hline Norvège & HRP à Halden & 58 & 19 \\
\hline France & OSIRIS & Mis à l'arrêt en 2015, après 49 années d'exploitation & 70 \\
\hline Suède & R2 à Studsvik & Mis à l'arrêt en 2005, après 45 années d'exploitation & 50 \\
\hline
\end{tabular}

Le projet $\mathrm{RJH}$ rassemble, autour du CEA, des instituts de recherche belge, tchèque, espagnol, finlandais et japonais, ainsi que les industriels EDF et AREVA (Framatome) en France et Vattenfall en Suède. En 2008, le Département atomique indien (DAE) a rejoint les sept partenaires initiaux du projet. Les accords signés entre ces différents partenaires leur donneront un accès à l'installation pour y mener leurs propres expérimentations.

L'objectif des promoteurs du projet RJH est de constituer une grande infrastructure européenne ouverte à la collaboration internationale pour contribuer à couvrir les besoins en matière de recherche et de développement pendant plusieurs décennies.

Le RJH est de type piscine à cœur fermé. Le cœur sera composé d'éléments combustibles de section circulaire, à plaques cintrées, refroidis par une circulation ascendante d'eau (circuit primaire). Cet ensemble sera entouré d'éléments réflecteurs (eau et béryllium) et placé dans une piscine. En termes de performances, la puissance maximale prévue est de $100 \mathrm{MW}$ et il est visé l'obtention d'un flux de neutrons de l'ordre de $10^{15}$ neutrons $/ \mathrm{cm}^{2} / \mathrm{s}$

137. Réacteur européen à eau sous pression. 
(énergie supérieure à $0,1 \mathrm{MeV}$ ) avec un flux de neutrons rapides d'énergie supérieure à $1 \mathrm{MeV}$ de l'ordre de $5.10^{14}$ neutrons $/ \mathrm{cm}^{2} / \mathrm{s}$. Le spectre de neutrons aura la particularité d'avoir deux bosses en termes d'énergie, permettant à la fois de mener des irradiations dans le domaine des neutrons thermiques (applications pour les réacteurs classiques actuels) et partiellement dans le domaine des neutrons rapides (applications pour les réacteurs à neutrons rapides de « quatrième génération $\left.{ }^{138} »\right)$.

Les dispositifs d'irradiation pourront être disposés au centre d'éléments combustibles (voir le schéma du bas de la figure 5.10), à la place d'éléments combustibles ou encore dans le réflecteur (figure 5.9). Des systèmes de déplacement situés en périphérie du réacteur permettront également de simuler des régimes transitoires représentatifs de situations transitoires, incidentelles ou accidentelles, susceptibles de survenir dans des réacteurs de puissance notamment (rampes lentes de puissance).

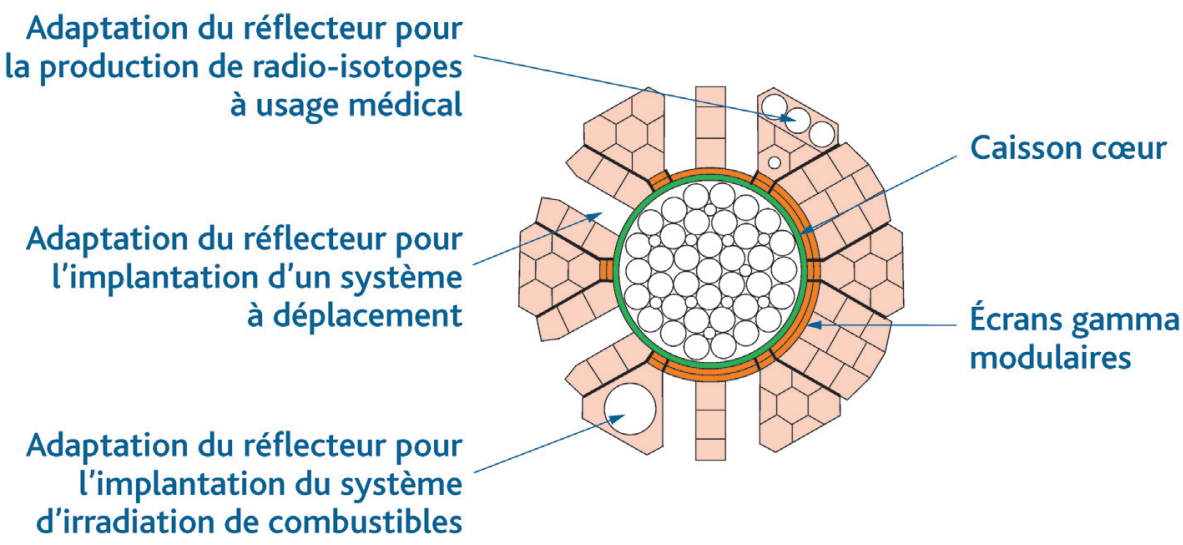

Figure 5.9. Implantations prévues dans la zone du réflecteur du réacteur Jules Horowitz. @ Georges Goué/IRSN.

La plupart des échantillons qui seront placés dans le cœur du RJH seront préparés et analysés dans les laboratoires d'étude des combustibles du centre CEA de Cadarache, situés à proximité du $\mathrm{RJH}$, ce qui réduira les transports de substances radioactives (et ainsi les risques associés).

Il est prévu que le RJH contribue pour $25 \%$ à la production européenne de radioisotopes à usage médical, voire, si besoin, jusqu'à $50 \%$. Après l'arrêt du réacteur OSIRIS, le technétium $99^{\mathrm{m}}$ est produit notamment dans les réacteurs de recherche HFR à Petten (Pays-Bas) et BR2 à Mol (Belgique) - le réacteur canadien NRU datant de 1957, qui produit plus de $40 \%$ de la production mondiale, a été remis en fonctionnement, après divers arrêts, notamment après la découverte en 2009 d'une fuite d'eau lourde située à la base de la cuve du réacteur. Ce réacteur a été arrêté définitivement au mois de mars 2018.

138. Le lecteur pourra consulter notamment l'ouvrage «Panorama des filières de réacteurs de quatrième génération (GEN IV) // Appréciations en matière de sûreté et de radioprotection ", Collection documents de référence, IRSN 2012/158, disponibles sur www.irsn.fr. 
En termes d'architecture générale, le RJH est constitué de deux bâtiments (voir la figure 5.10) :

- le bâtiment du réacteur, qui abrite le réacteur lui-même, les équipements nécessaires à son exploitation et ceux utilisés pour suivre les expérimentations (postes de travail pour les expérimentateurs, équipements électriques, de contrôle-commande, etc.) ;

- le bâtiment des annexes nucléaires, qui comporte des cellules pour la préparation, le conditionnement et l'examen des échantillons expérimentaux et trois piscines d'entreposage.

Ces deux bâtiments sont posés sur un seul et même radier ; ils constituent « l'unité nucléaire ». Une isolation parasismique est réalisée par des plots. Elle sera précisée au paragraphe 7.4 .2

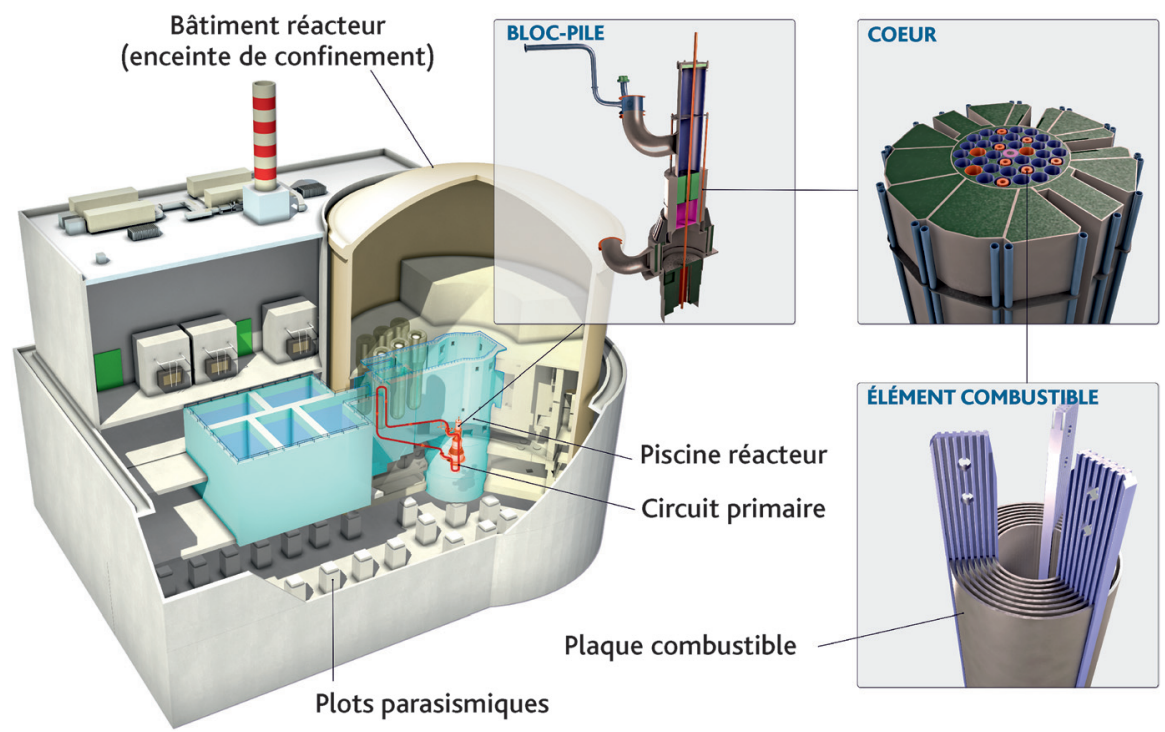

Figure 5.10. Réacteur Jules Horowitz : schémas de l'unité nucléaire, du bloc-pile, du cœur et d'un élément combustible. @ A ASN.

Le réacteur est équipé de trois circuits :

- le circuit primaire qui refroidit le cœur du réacteur par circulation ascendante d'eau sous pression (une dizaine de bars à l'entrée du cœur). Ce circuit fermé est situé à l'intérieur du bâtiment du réacteur. Le cœur du réacteur et une partie du circuit primaire sont immergés dans la piscine du réacteur (figure 5.11) ;

- le circuit secondaire, isolé du circuit primaire, qui refroidit le circuit primaire grâce à des échangeurs de chaleur placés entre les deux circuits dans le bâtiment du réacteur. La pression du circuit secondaire sera plus élevée que celle du circuit primaire, pour que notamment, en cas de fuite entre le circuit primaire et le circuit secondaire, une contamination ne puisse affecter l'eau du circuit secondaire ; 

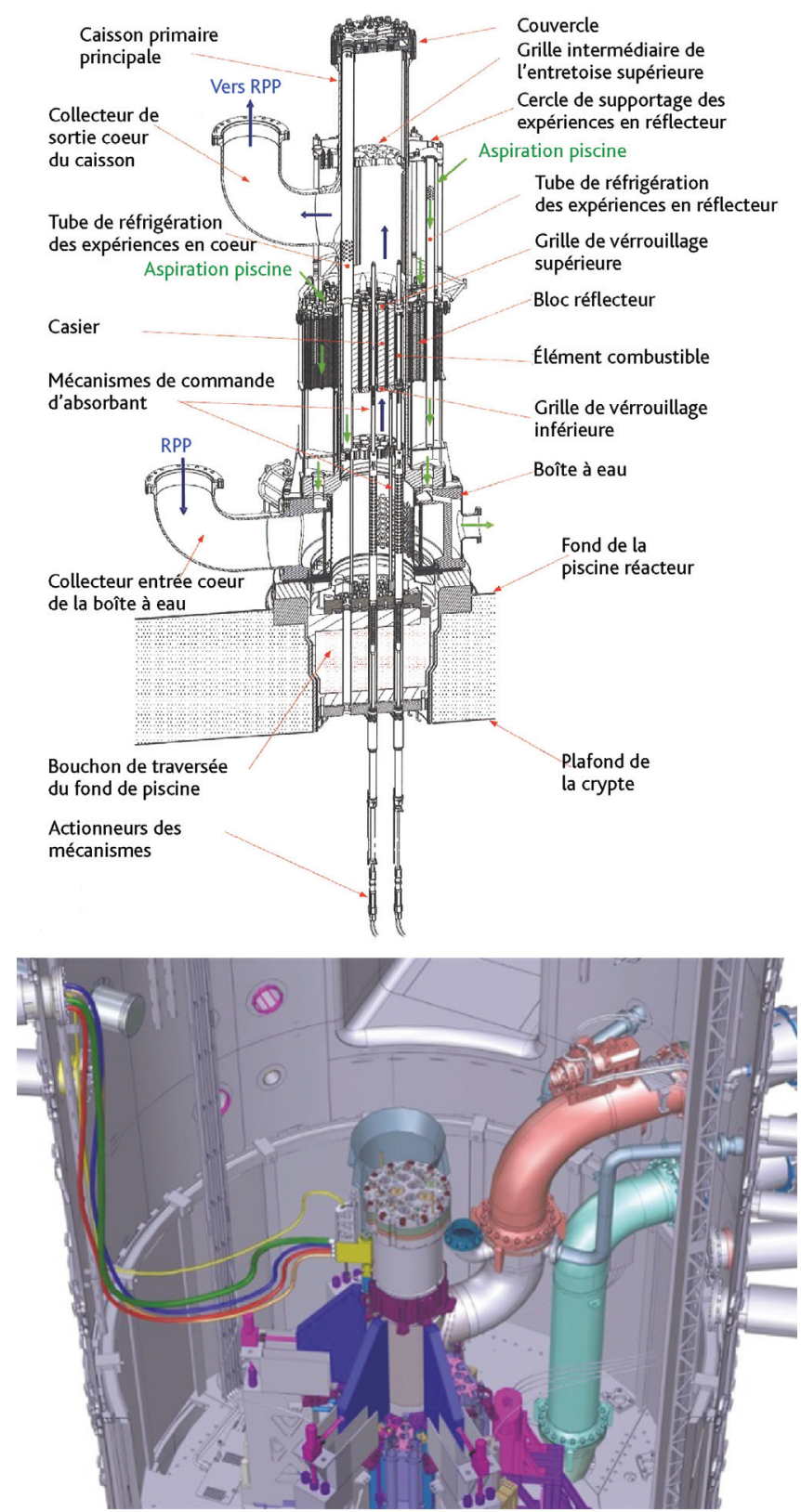

Figure 5.11. Le bloc-pile ${ }^{139}$ du réacteur Jules Horowitz. (C) DR.

139. Il s'agit d'une expression utilisée pour les réacteurs de recherche, l'expression bloc-réacteur étant plutôt utilisée pour les réacteurs de puissance. 
- le circuit externe qui refroidit l'eau du circuit secondaire à travers d'autres échangeurs de chaleur placés dans un autre bâtiment de l'installation (bâtiment des réfrigérants). Ce circuit sera connecté par une canalisation au canal de Provence. L'eau sera restituée au canal EDF, de telle sorte que l'eau de ce canal reste inférieure à $25^{\circ} \mathrm{C}$ conformément à la règlementation.

Ces trois circuits en série vont permettre de réduire le risque de transfert de contamination du cœur vers l'environnement (l'eau du canal EDF).

L'installation RJH dispose de trois piscines d'entreposage :

- la piscine d'entreposage des combustibles usés, qui servira à l'entreposage des combustibles usés du réacteur avant leur traitement à l'usine Cogema de La Hague ;

- la piscine d'entreposage des dispositifs irradiés, qui permettra d'entreposer des dispositifs expérimentaux (une trentaine) et d'effectuer des examens sous eau ;

- la piscine d'entreposage des composants irradiés et de démantèlement, qui permettra d'entreposer des composants des structures internes du réacteur, des structures du cœur (réflecteur) et les outillages utilisés pour la manutention et le démontage.

Les éléments combustibles et les dispositifs expérimentaux seront transférés sous eau entre le réacteur et les piscines ou les cellules.

Les objectifs généraux de sûreté retenus pour la conception du $\mathrm{RJH}$, en termes de conséquences radiologiques d'incidents et d'accidents, sont similaires à ceux adoptés pour les réacteurs électronucléaires de nouvelle génération tels qu'EPR ; ce point sera précisé au chapitre 7.

Les rejets gazeux liés à l'installation $\mathrm{RJH}$ seront générés principalement lors de l'ouverture des dispositifs expérimentaux en cellule et lors des opérations de dégazage du circuit primaire. Les systèmes de ventilation seront équipés de filtres à très haute efficacité (THE) et de pièges à iode (PAI) permettant de limiter la quantité des rejets gazeux. Il est visé que l'activité de ces rejets - composés principalement de gaz rares (xénon, krypton...), d'halogènes (iode...) et de tritium - soit similaire à celle mesurée sur les réacteurs de recherche existants, en tenant compte des expériences qui y seront menées.

Concernant les rejets liquides, ils devraient être générés principalement lors des expérimentations, des opérations de décontamination des cellules et de la régénération des résines utilisées pour l'épuration de l'eau de la piscine du réacteur et des piscines d'entreposage. Ces effluents radioactifs seront pris en charge par l'unité de traitement du centre de Cadarache.

Les estimations faites par le CEA font apparaître que du fait de l'exploitation du RJH, les rejets gazeux et liquides du centre de Cadarache devraient rester de l'ordre de quelques pourcent des valeurs stipulées dans les autorisations annuelles fixées pour ce centre.

L'autorisation de création de l'installation RJH a été prononcée par décret ${ }^{140}$ en 2009 (sa mise en service n'est pas envisagée avant 2020).

140. Décret $n^{\circ} 2009-1219$ du 12 octobre 2009. 
Parmi ses réacteurs en fonctionnement, la France compte aussi deux réacteurs délivrant des faisceaux de neutrons utilisés à des fins de recherche scientifique. Il s'agit respectivement du réacteur ORPHEE, exploité par le CEA à Saclay ${ }^{141}$, et du réacteur à haut flux (RHF) exploité par l'Institut Laue-Langevin à Grenoble.

Ces réacteurs fonctionnent par cycles de plusieurs semaines, séparés par des périodes d'arrêt pour le chargement de combustible et pour les travaux de maintenance. Dans ces réacteurs, de l'eau lourde est utilisée pour ralentir les neutrons produits par les fissions (modération neutronique) et les rendre utilisables pour l'étude de la matière :

- dans le réacteur RHF, de l'eau lourde sert de fluide caloporteur du cœur (constitué par un élément combustible), lui-même placé dans une cuve d'eau lourde, le tout disposé dans une piscine d'eau légère ;

- dans le réacteur ORPHEE, le rôle de fluide caloporteur du cœur est assuré par de l'eau légère, l'eau lourde servant de réflecteur, le tout disposé dans une piscine d'eau légère (voir la figure 5.12).

L'énergie des neutrons devant être modulée selon les besoins des expériences, des dispositifs spécifiques sont utilisés pour modifier localement cette énergie. Ainsi, un récipient rempli d'hydrogène ou de deutérium liquide (à une température de l'ordre de - $250^{\circ} \mathrm{C}$ ) permet d'obtenir des neutrons lents (" source froide ${ }^{142}$ »), et un bloc de graphite chauffé à plus de $1000{ }^{\circ} \mathrm{C}$ permet d'obtenir des neutrons de forte énergie (« source chaude »).

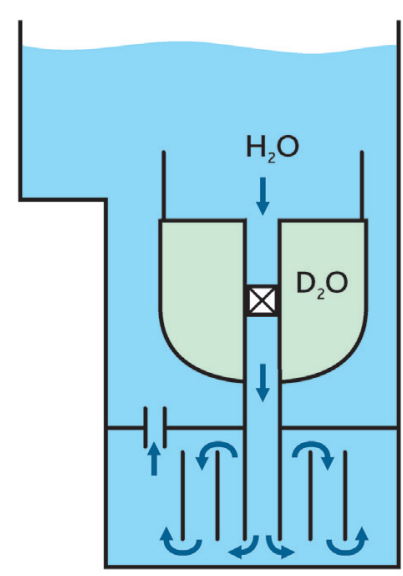

ORPHÉE

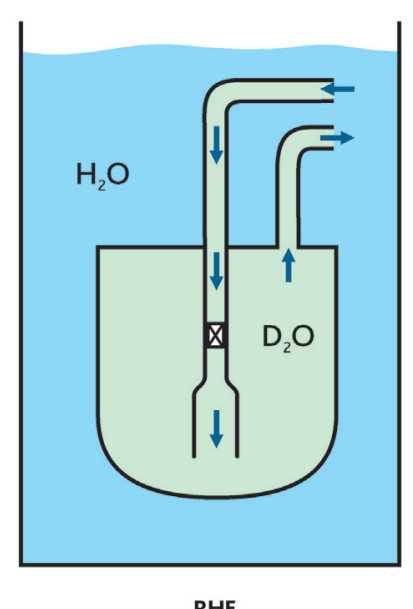

RHF

Figure 5.12. Utilisations respectives de l'eau légère et de l'eau lourde dans les réacteurs ORPHEE et RHF. Dans les deux cas, l'eau circule de façon descendante dans le cœur. (c) Georges Goué/IRSN.

141. Il est à noter que l'installation nucléaire de base correspondante (INB $\left.n^{\circ} 101\right)$ comprend le réacteur ORPHEE et le Laboratoire Léon Brillouin (LBB) qui rassemble des chercheurs du CNRS et du CEA dans le domaine de la spectrométrie neutronique.

142. À ne pas confondre avec la source externe de refroidissement d'un réacteur (eau d'une rivière, air...). 

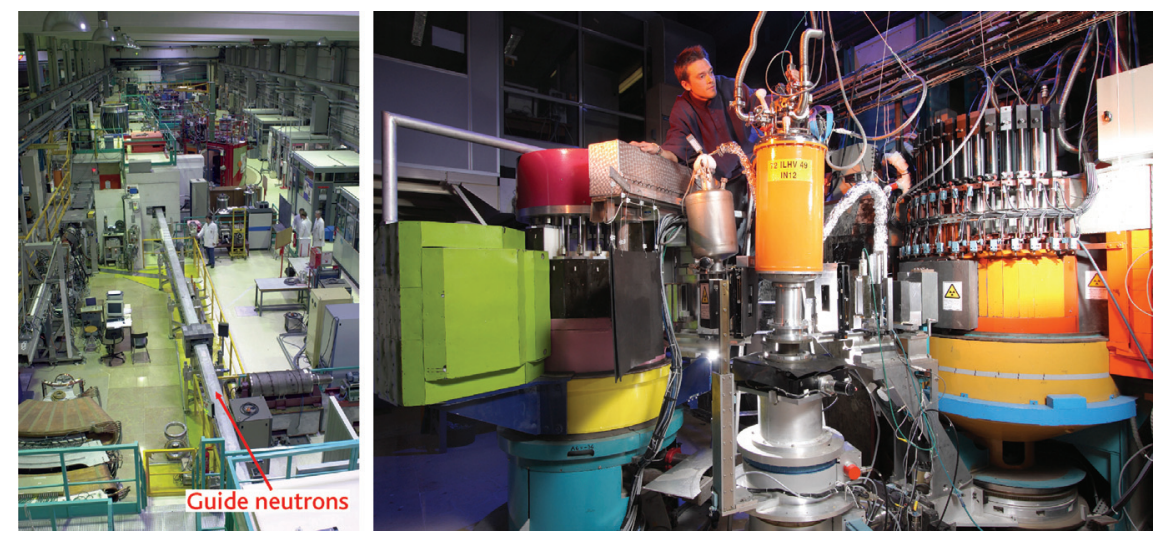

Figure 5.13. À gauche, un hall de guides de neutrons (RHF). @ Artechnique/ILL; à droite, diffractomètre D10. (๐ ILL.

La « collecte » des neutrons (thermiques, lents ou rapides) pour leurs utilisations expérimentales s'effectue via le « nez » de canaux (extrémités des « doigts de gant ») orientés vers le cœur du réacteur. Les neutrons ainsi collectés sont ensuite " guidés » jusqu'à l'extérieur de la piscine où un filtre permet de prélever les neutrons dont la longueur d'onde est adaptée à l'irradiation recherchée de l'échantillon de matière étudiée (dans « l'aire expérimentale » ou « hall des guides de neutrons » situé autour du réacteur - figure 5.13). Les autres neutrons du faisceau terminent leur course dans un mur de béton servant de protection.

Ces réacteurs disposent également de canaux verticaux à proximité de la cuve d'eau lourde, utilisés principalement à des fins d'irradiation.

Ainsi, le réacteur ORPHEE est équipé de neuf doigts de gant horizontaux (multifaisceaux), tangentiels au cœur, permettant l'utilisation de 20 faisceaux de neutrons (figure 5.14). Le nez des doigts de gant est situé dans le modérateur (eau lourde) à proximité du cœur, là où le flux des neutrons thermalisés est maximal ; trois nez visent deux " sources froides », deux autres une " source chaude ». Les physiciens qui effectuent des recherches autour d'ORPHEE appartiennent à un laboratoire commun au CEA et au CNRS, le Laboratoire Léon Brillouin (LLB), et travaillent dans des domaines aussi variés que la chimie, la biologie, la métallurgie et la physique. Le réacteur ORPHEE permet par ailleurs d'effectuer des analyses par activation en partenariat avec le Laboratoire Pierre Süe, d'irradier des échantillons et de produire des radioisotopes à usage industriel et médical (Cis-bio international), et enfin de doper des monocristaux de silicium par transmutation nucléaire.

D'une puissance maximale de $14 \mathrm{MW}$, le réacteur ORPHEE (figure 5.15) délivre un flux de neutrons pouvant atteindre $3.10^{14}$ neutrons $/ \mathrm{cm}^{2} / \mathrm{s}$ dans la cuve d'eau lourde. Son cœur, composé de huit éléments combustibles de section carrée et à plaques planes, dont l'uranium métallique est fortement enrichi en isotope 235 (93\%), est refroidi par de l'eau légère circulant à $7,5 \mathrm{~m} / \mathrm{s}$. L'ensemble du cœur du réacteur et de la cuve d'eau lourde (en acier inoxydable) est plongé dans une piscine d'eau légère. 


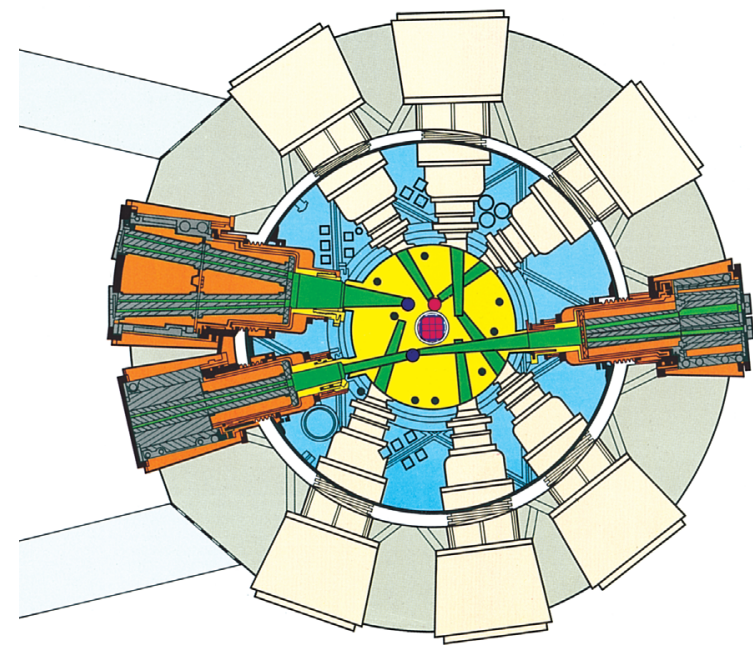

Figure 5.14. Coupe horizontale du réacteur ORPHEE montrant les neuf doigts de gant des canaux neutroniques. Au centre le cœur, les points rouges désignent les « sources froides » et la « source chaude »; l'eau lourde est représentée en jaune, l'eau légère en bleu. (c) CEA.

Le réacteur à haut flux (RHF, figure 5.16), lieu de recherche international, est équipé de 13 doigts de gant horizontaux, quatre doigts de gant inclinés et deux doigts de gant verticaux (l'un des deux étant utilisé pour la source neutronique de démarrage du réacteur). Il peut délivrer simultanément des neutrons dans une quarantaine de dispositifs de recherche différents. Neuf doigts de gant visent deux " sources froides », quatre doigts de gant visent la « source chaude ». Le cœur du RHF est constitué d'un

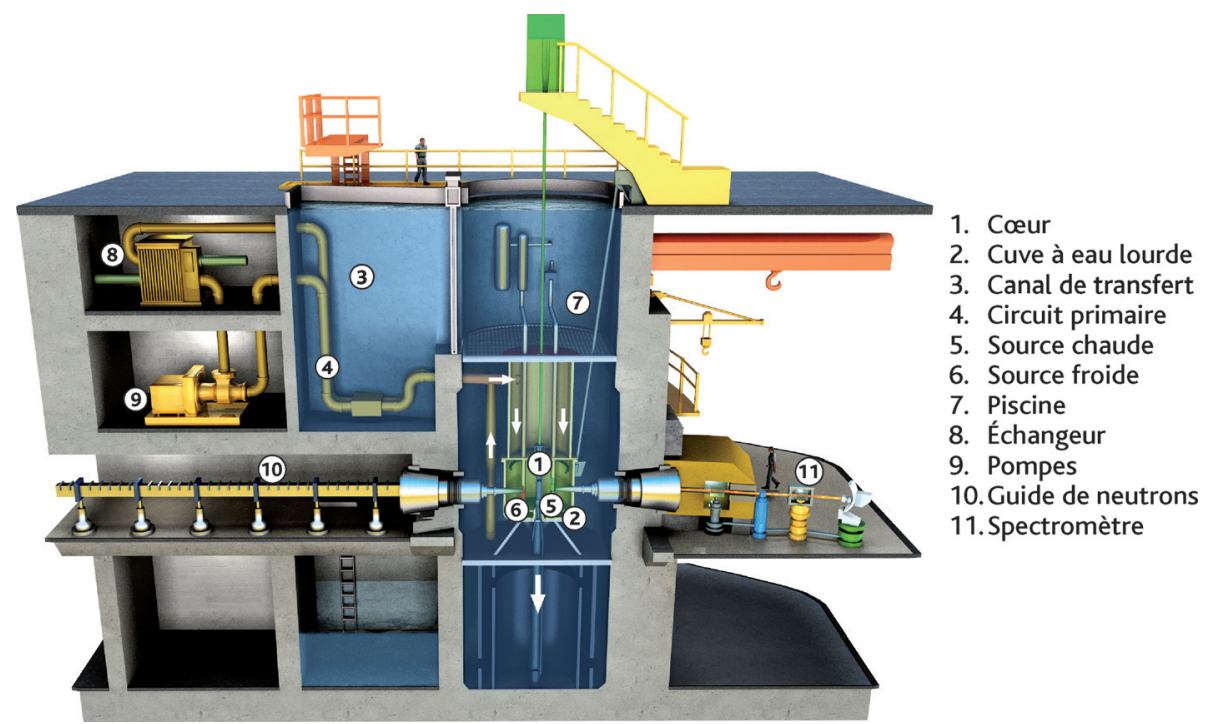

Figure 5.15. Réacteur ORPHEE : schéma du bloc-pile, coupe verticale. (c) CEA. 


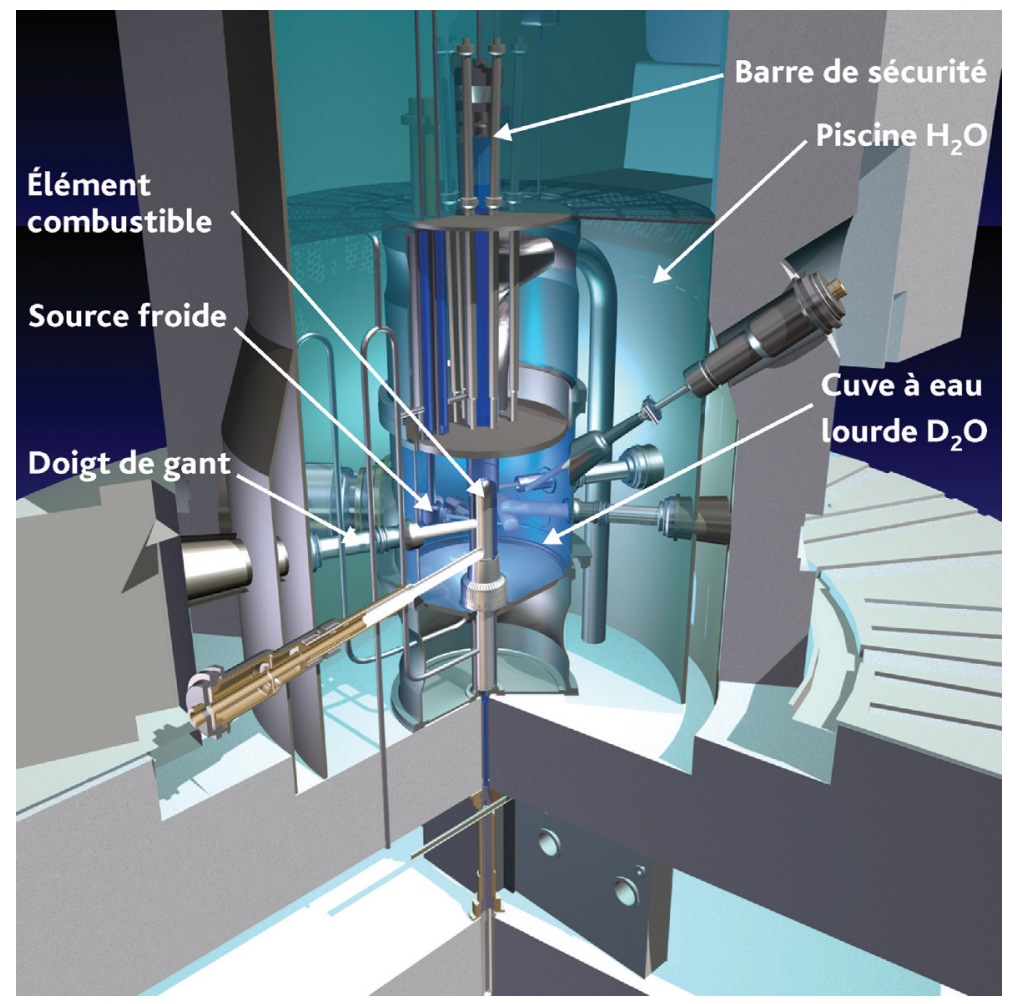

Figure 5.16. Schéma du RHF. (c) ILL.

unique élément combustible de forme annulaire à plaques cintrées, dont l'uranium métallique est fortement enrichi en isotope 235 (93\%). Il est refroidi à l'eau lourde, circulant à la vitesse de $5,5 \mathrm{~m} / \mathrm{s}$. Il permet de produire le flux de neutrons le plus intense d'Europe, à ce jour, soit $1,5.10^{15}$ neutrons $/ \mathrm{cm}^{2} / \mathrm{s}$ (flux de neutrons thermique dans la cuve d'eau lourde). Comme dans le cas d'ORPHEE, l'ensemble du cœur du réacteur et de la cuve d'eau lourde (en alliage d'aluminium dit AGBNET dans le cas du RHF) est plongé dans une piscine d'eau légère.

Il est à noter que l'installation de détritiation de l'eau lourde, qui était située à proximité de l'ILL, a été définitivement arrêtée, l'exploitant ayant décidé de confier la détritiation de l'eau lourde à une entreprise canadienne.

En 2016, la France exploite encore un réacteur de recherche entièrement dédié à des essais dans le domaine de la sûreté, plus précisément à l'étude du comportement d'éléments combustibles de réacteurs électronucléaires à eau dans certaines situations accidentelles. Il s'agit du réacteur CABRI (figure 5.17), implanté au centre CEA de Cadarache, qui permet de soumettre un échantillon de combustible nucléaire, neuf ou irradié, aux conditions qui résulteraient d'un accident de réactivité. Pour ce faire, le réacteur est constitué d'un cœur nourricier et d'une boucle expérimentale, dont la partie située au centre du cœur nourricier reçoit le dispositif d'essai qui contient le combustible 


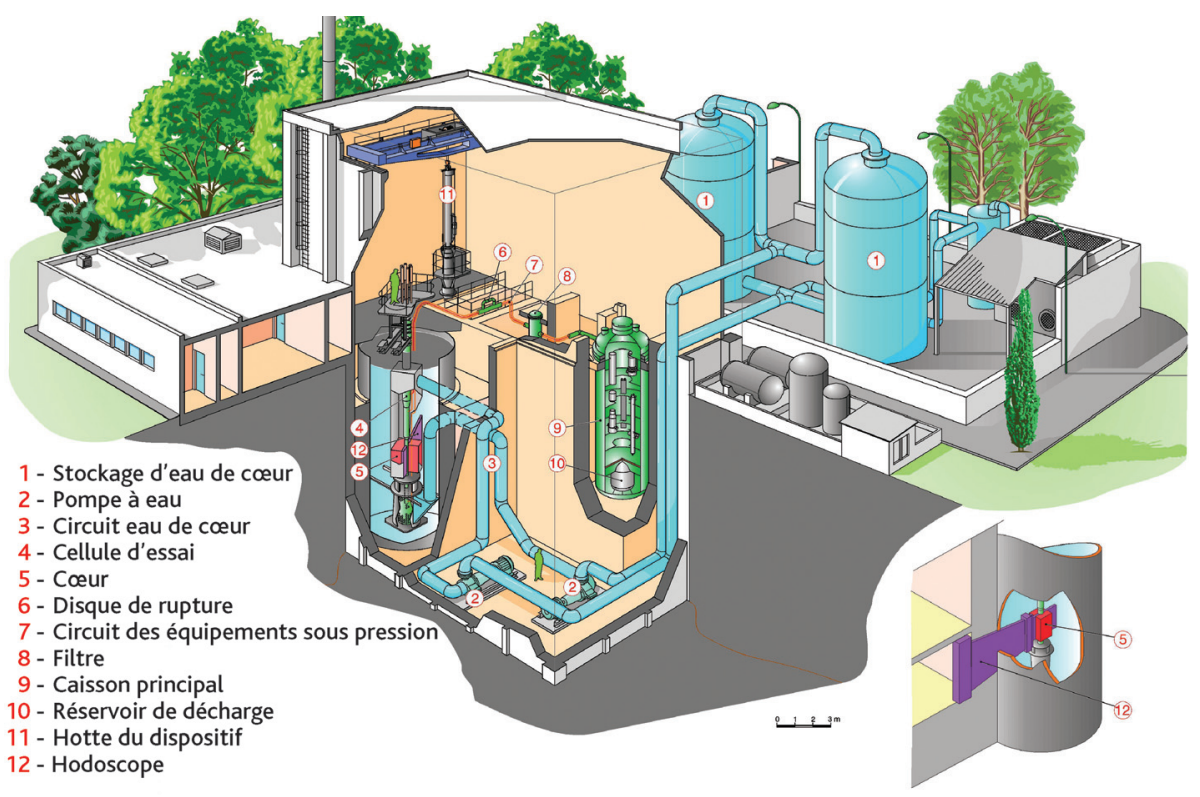

Figure 5.17. Schéma du réacteur CABRI et de sa boucle expérimentale à eau sous pression. @ DPAM/IRSN.

à tester. Le cœur nourricier fournit le flux neutronique nécessaire pour obtenir la puissance désirée dans le combustible soumis à un essai tandis que la boucle expérimentale permet de le soumettre à des conditions thermohydrauliques représentatives de celles d'un réacteur de puissance. La particularité de ce réacteur réside dans le système d'injection contrôlée de réactivité. À cet effet, quatre assemblages du cœur nourricier sont équipés, en lieu et place de la dernière couronne de crayons de combustible, de tubes périphériques cylindriques ${ }^{143}$ qui sont remplis d'hélium 3 sous pression avant le démarrage du réacteur (ce gaz absorbe fortement les neutrons). Une fois la puissance nominale atteinte dans le réacteur et les conditions thermohydrauliques requises obtenues dans la boucle expérimentale, ces tubes sont dépressurisés grâce à l'ouverture de vannes selon une séquence déterminée, ce qui modifie de façon plus ou moins rapide la réactivité ${ }^{144}$ et donc la puissance délivrée par le cœur nourricier. Pour l'étude d'injections de réactivité très rapides, il est possible d'accroître la puissance du réacteur CABRI de $100 \mathrm{~kW}$ à $20 \mathrm{GW}$ en quelques millisecondes ; la puissance retombe ensuite tout aussi rapidement du fait des effets de contre-réaction neutronique (effet Doppler en particulier).

Dans le passé, le réacteur CABRI a été équipé d'une boucle en sodium qui a servi à divers programmes jusque dans les années 2000 - non seulement pour les réacteurs à neutrons rapides refroidis par du sodium mais aussi pour les réacteurs à eau sous pression (essais dits REP-Na). Cette boucle a été remplacée par la boucle à eau sous pression.

143. L'expression « barres transitoires » est utilisée.

144. Le gaz disparaissant du cœur, le nombre de neutrons dans le cœur augmente, provoquant davantage de fissions. 
\#FOCUS

\section{Quelques aspects de sûreté de réacteurs électronucléaires explorés dans le cadre de programmes expérimentaux menés dans les réacteurs de recherche français}

Les réacteurs de recherche constituent des outils indispensables à la recherche scientifique et technologique et à l'accompagnement du développement des réacteurs de puissance (réacteurs électronucléaires). Les expériences qui y ont été menées ont permis notamment d'accroître la sûreté des réacteurs de puissance dans le domaine des accidents, par une meilleure connaissance des phénomènes mis en jeu.

Le réacteur OSIRIS a été utilisé pour l'étude du comportement de gaines de combustibles de réacteurs à eau sous pression soumis à une augmentation lente de puissance (rampes lentes, de quelques dizaines de secondes à quelques minutes) : de tels essais ont été réalisés sur des tronçons de crayons de combustible irradiés et ont permis d'établir des limites d'utilisation pour différents matériaux de gainage.

Des programmes d'essais réalisés dans le réacteur CABRI ont permis d'étudier le comportement d'éléments combustibles des réacteurs de puissance en cas de retrait ou d'éjection d'éléments absorbants des cœurs de ces réacteurs ${ }^{145}$.

Ainsi, de 1978 à 2001, le réacteur CABRI a été utilisé pour l'étude de l'accident de retrait intempestif d'une barre de commande (RIB) dans les réacteurs à neutrons rapides (RNR) refroidis par du sodium, par des essais (de type rampe lente) réalisés avec des aiguilles ${ }^{146}$ de combustible uniques positionnées dans une boucle remplie de sodium. Le risque d'éjection de combustible fondu hors d'une aiguille en cas de défaut préexistant de gainage a notamment été étudié.

Il est à noter que le réacteur SCARABEE a également été utilisé, de 1983 à 1989, pour l'étude, grâce à une boucle en sodium de plus grand diamètre que celle utilisée dans CABRI, des accidents hypothétiques de bouchage et de fusion d'assemblages dans les RNR. Les essais ont été menés sur de petits assemblages comportant jusqu'à 37 aiguilles.

La boucle de sodium de réacteur CABRI a aussi été utilisée pour l'étude du comportement de crayons de combustible des réacteurs à eau sous pression (REP) en cas d'éjection d'une grappe absorbante. Cet accident suppose la rupture du mécanisme de la grappe. L'éjection résulte de la différence de pressions qui existe entre le circuit primaire et l'enceinte de confinement. Cette éjection violente entraîne un emballement local de la réaction nucléaire pendant quelques dizaines de millisecondes («pulse » de puissance) provoquant une augmentation rapide de

145. Voir l'ouvrage «État des recherches dans le domaine de la sûreté des réacteurs à eau sous pression », Collection sciences et techniques, IRSN/EDP Sciences, 2017.

146. Expression utilisée pour les RNR, l'expression crayon étant celle utilisée pour les REP. 
la température du combustible. Les contre-réactions neutroniques limitent le transitoire de puissance avant l'arrêt d'urgence du réacteur qui intervient dans un second temps. L'échauffement brutal des pastilles de combustible lors du « pulse » de puissance entraîne leur dilatation rapide et, dans certains cas, un relâchement de gaz de fission présents dans le combustible. Soumise à ces contraintes, la gaine du crayon combustible peut se rompre, libérant des fragments de combustible extrêmement chauds. Pour les réacteurs à eau sous pression, des critères ont été établis dans les années 1970 sur la base des résultats des essais SPERT $^{147}$ menés aux États-Unis avec du combustible frais ou peu irradié. Les essais menés dans les années 1990 dans le réacteur CABRI (ainsi que dans le réacteur NSRR au Japon) sur des tronçons de crayons industriels préirradiés dans les réacteurs à eau sous pression ont permis d'étudier les phénomènes mécaniques qui se produisent dans les premières dizaines de millisecondes de l'excursion de puissance, pendant lesquelles la température de la gaine est peu affectée : il s'agit des essais dits REP-Na, incluant notamment un essai sur du combustible MOX ${ }^{148}$ ayant atteint un taux de combustion de $55 \mathrm{GWj} / \mathrm{tU}$, et un essai avec un crayon gainé avec un nouvel alliage, dit $\mathrm{M} 5^{\circledR}$.

Pour étudier les phénomènes qui se produiraient après les premières centaines de millisecondes (assèchement et gonflement des gaines), ainsi que les conséquences en termes d'onde de pression d'une éventuelle dispersion du combustible dans le réfrigérant, l'IRSN a prévu de nouveaux essais dans la boucle à eau sous pression du réacteur CABRI - il s'agit du projet de l'OCDE $^{149} / \mathrm{AEN}$ dénommé Cabri International Programme (CIP), mené en partenariat avec EDF et de nombreux organismes de sûreté et industriels étrangers.

Le réacteur PHEBUS a grandement contribué à l'acquisition de connaissances sur les accidents de fusion du cœur d'un $\mathrm{REP}^{150}$. Le programme international Phébus-PF (PF pour produits de fission), piloté par l'IRSN, a permis de simuler à une échelle réduite la fusion du cœur. L'objectif principal de ce programme était de contribuer à l'amélioration des connaissances sur les rejets radioactifs qui pourraient se produire dans l'environnement lors d'un tel accident. À cet effet, cinq essais ont été réalisés de 1993 à 2004 dans un dispositif d'essai implanté dans le réacteur PHEBUS. Ces essais visaient à reproduire les phénomènes physiques majeurs qui gouvernent la fusion d'un cœur de réacteur à eau sous pression ainsi que les transferts des substances radioactives depuis le combustible nucléaire jusqu'à l'enceinte de confinement. Le dispositif (figure 5.18), qui permettait de simuler le cœur, le circuit primaire de refroidissement et l'enceinte

147. SPERT (Special Power Excursion Reactor Test) est le nom donné à des réacteurs d'expérimentation dit d'excursion de puissance du centre d'essais d'Idaho Falls aux États-Unis.

148. Mixed Oxide Fuel (combustible mixte $\mathrm{UO}_{2}+\mathrm{PuO}_{2}$ ).

149. Organisation de coopération et de développement économique.

150. Voir ici aussi l'ouvrage « État des recherches dans le domaine de la sûreté des réacteurs à eau sous pression », Collection sciences et techniques, IRSN/EDP Sciences, 2017, ainsi que l'ouvrage « Les accidents de fusion du cœur des réacteurs nucléaires de puissance - État des connaissances », Collection sciences et techniques, IRSN/EDP Sciences, 2013. 


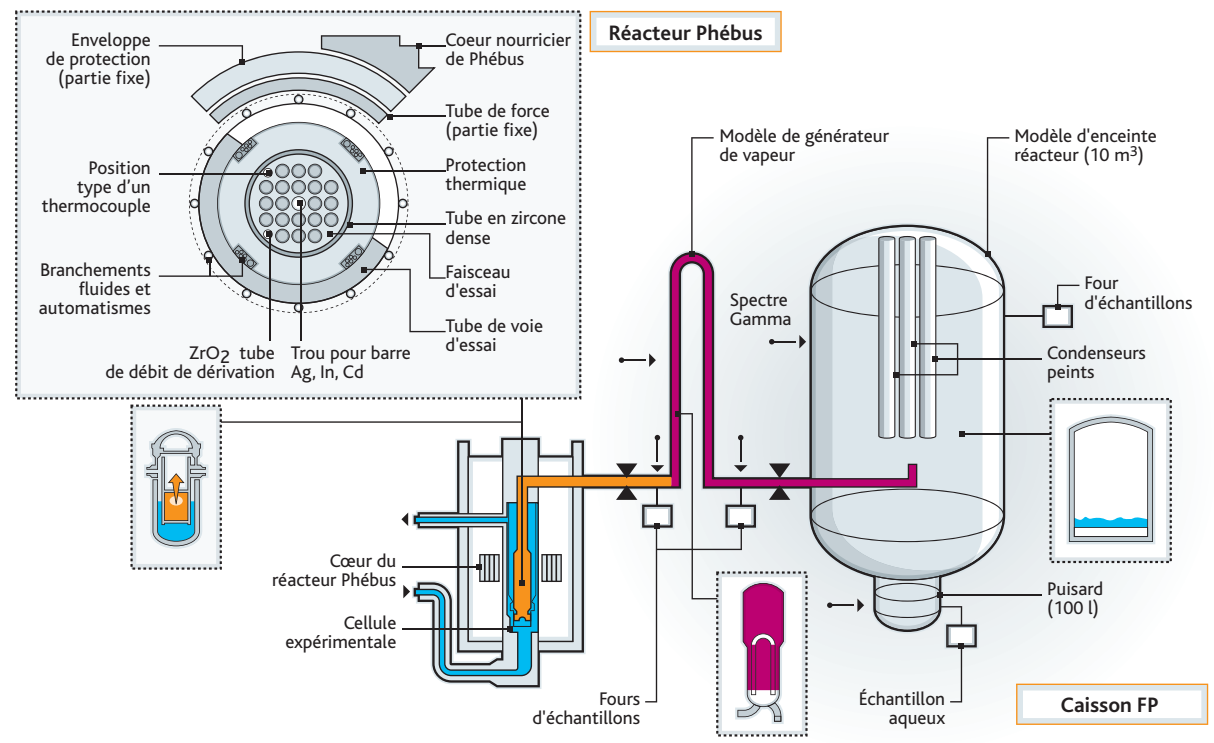

Figure 5.18. Schéma de l'installation Phébus-PF. C Stéphane Jungers/IRSN - Source IRSN.

de confinement, était constitué d'une partie « en pile " passant dans le cœur du réacteur PHEBUS et d'une partie " hors pile » appelée "caisson PF ». Le combustible d'expérimentation, préalablement irradié dans un réacteur de recherche ou dans un réacteur électronucléaire, était introduit dans la partie " en pile » du dispositif. Plus de 200 capteurs de mesure et environ 100 dispositifs de prélèvement constituaient l'instrumentation de l'essai. Après un fonctionnement en puissance du réacteur PHEBUS, destiné à recréer dans l'assemblage d'essai des produits de fission à vie courte, tels que l'iode 131, la puissance était abaissée et le refroidissement de l'assemblage d'essai était arrêté. Le combustible d'essai s'échauffait alors rapidement jusqu'à la fusion. Les produits de fission qui s'échappaient des gaines du combustible étaient dirigés vers le caisson expérimental. Les expériences réalisées ont permis d'obtenir des données majeures pour la compréhension des mécanismes de dégradation du cœur d'un REP ainsi que du comportement des produits de fission relâchés dans les circuits. Ces essais ont également confirmé des connaissances déjà acquises par des expériences à l'échelle du laboratoire et ont permis d'améliorer et de valider plusieurs logiciels de simulation d'accidents de fusion du cœur développés et utilisés dans le monde, dont certains utilisés par l'IRSN pour ses expertises de sûreté ou pour ses études probabilistes de sûreté de niveau 2 (logiciel ASTEC ${ }^{151}$ ).

151. Accident Source Term Evaluation Code (logiciel de simulation pour l'évaluation des phénomènes physiques intervenant au cours d'un accident de fusion du cœur d'un réacteur à eau sous pression). 
